J. Environ. Sci.

Institute of Environmental Studies and Research - Ain Shams University

\title{
ENVIRONMENTAL RISKS ASSESSMENT FOR SOILS AND PLANTS IRRIGATED FROM EI-MARIOUTEYA AND EL-RAHAWY DRAIN
}

Aya A. Taha ${ }^{(1)}$; Esam A. Kishar ${ }^{(2)}$ and Abd El-Hamid A. A. Sherif ${ }^{(3)}$

1) Egyptian Environmental Affairs Agency, El-Maadi, Cairo, Egypt 2) Chemistry Dep. Women's College, Ain Shams Univ., Cairo, Egypt. 3) Enviro. Rese. Dep. Soils, Water \& Envir. Res. Instit., Giza, Egypt

\begin{abstract}
The objective of this study was to evaluate the environmental risks of water, soils, and plants from the El-Mariouteya Canal and El-Rahawy drain. To achieve this aim, $\mathrm{pH}$ and electrical conductivity EC in water and soils, as well as sodium adsorption ratio (SAR) in water; some micro elements and heavy metals were estimated in water; soils and plants. In addition to calculation of contamination factor $(\mathrm{CF})$, degree of contamination $(\mathrm{Cd})$, modified degree of contamination (mCd); pollution load index (PLI) and bioaccumulation factor of elements in plants. The obtained results indicated that the values of $\mathrm{pH}$ and (SAR) in the water were different from season to another season. (EC) values were very high and unsuitable for irrigation in summer season; while they were suitable for irrigation in winter season according to FAO. Regard to soil $\mathrm{pH}$ values tend to be normal, slightly and moderately alkaline and (EC) was ranged between non-saline to highly saline soils. However, boron, cadmium, cobalt, chromium, nickel and lead were within the safe limits. The total content of cadmium exceeded the safe limits in both seasons, but other elements were within the safe limits.

The lowest concentration of iron, boron, cobalt and chromium was found in Wheat yield. Manganese and nickel were found in the fruits of Eggplant, zinc and copper were found in Cabbage. While the highest concentrations of iron, manganese, copper, cobalt, chromium and nickel were found in Okra. Zinc was found in Spinach and boron was found in Watercress. BCF indicates that the most plants have more than 1 and showed high concentrations of heavy metals. The values of (CF) were low for both zinc, boron and lead for
\end{abstract}


all sites and medium for copper and cobalt at all sites and low to medium with both iron, manganese, chromium and nickel indicating that this contamination is related to human activities. Modified contamination degree $(\mathrm{mCd})$ is moderate to high in some sites. The (PLI) is generally high $(>1)$ in all sites; exception one site. The (PLI) was low $(<1)$ in some sites in winter season.

Keywords: water, soil, plant, pollution, bioaccumulation and risk assessment.

\section{INTRODUCTION}

El-Moheet drain receives all waste water e.g. agricultural, domestic, and sewage from lateral minor drains. The maximum industrial units in the selected areas also discharge their effluents directly into El Moheet drain and the seepage taking from the effluents as well as other anthropogenic activities impair the quality of surface and ground water and making them unfit for irrigation purposes (Mohamed. 2014). The values of the detected heavy metals in El-Rahawy drain are appreciably higher than those in the River Nile water. The mean values of the elements at different sites showed that Fe to be the most abundant element in water whereas $\mathrm{Cd}$ got the least concentration (Gaber et al., 2013).

The application of wastewater increased the soil salinity, available microelements and decreased the soil $\mathrm{pH}$. The accumulation of micronutrients and heavy metals from wastewater application could be caused directly by the wastewater composition or indirectly through increasing solubility of the indigenous insoluble soil heavy metals as a result of the chelation or acidification action of the applied wastewater (Rusan et al., 2007). The heavy metals may adversely affect soil ecology, agricultural production or product quality, and ground water quality, and will ultimately harm to health of living organism by food chain (Ene et al, 2009). Elevated levels of heavy metals in 
J. Environ. Sci.

Institute of Environmental Studies and Research - Ain Shams University

irrigation water led to significantly higher concentrations of heavy metals in the soil compared to those obtained from clean water irrigated site (Singh $e t$ al., 2010).

Plant species have a variety of capacities to remove and accumulate heavy metals. The certain species may accumulate specific heavy metals, causing a serious risk to human health when plant - based foodstuffs are consumed (Fytianos et al., 2001). The application of wastewater to soil increased the yield and the $\mathrm{N}, \mathrm{P}, \mathrm{K}, \mathrm{Fe}, \mathrm{Mn}, \mathrm{Zn}, \mathrm{Cu}, \mathrm{B}$ and Mo contents of Cabbage plants without causing undesirable side effects to the plants heavy metal contents (Kiziloglu et al., 2007).

The leafy vegetables, such as Cauliflower, Cabbage and Spinach, grow quite well in the presence of sewage water, whereas other vegetables, such as Radish, are sensitive to sewage water (Kapourchal et al., 2009). The bioaccumulation of $\mathrm{Pb}$ and $\mathrm{Cr}$ in vegetables was above the critical concentrations of plant growth, while $\mathrm{Pb}$ and $\mathrm{Cd}$ were above the prescribed limit for animal diets (Khan et al., 2012). The concentrations of heavy metals in edible part of Spinach vary from metal to metal. The trend of accumulation in the Spinach showed an order of decreasing magnitude from $\mathrm{Fe}$ to $\mathrm{Cd}(\mathrm{Fe}>$ $\mathrm{Cu}>\mathrm{Mn}>\mathrm{Pb}>\mathrm{Cd}$ ). Iron had the highest content in Spinach sample with cadmium being the lowest of all the metals analyzed (Mustapha and Adeboye. 2014).

The overall contamination of soils based on the CF values which indicate that soils were considerably contaminated with $\mathrm{Fe}, \mathrm{Mn}, \mathrm{Pb}$, and $\mathrm{Zn}$, but showed signs of low contamination with Co. in the case of degree of contamination, the windward soils fall under considerable contamination. The 
modified degree of contamination suggest that the studied area is moderately contaminated $(\mathrm{mCd}=2.3)$ (Likuku et al., 2013). The soil samples were moderately contaminated with $\mathrm{Mn}, \mathrm{Cu}$ and $\mathrm{Ni}$ while $\mathrm{Cr}, \mathrm{Pb}$ and $\mathrm{Zn}$ showed low contamination factor. $70 \%$ of the samples showed low degree of contamination while $30 \%$ indicated moderate degree of contamination (Omotoso and Ojo. 2015). The calculated (PLI) values of metals were ranged from 1.36 to 2.07 during summer and 1.83 to 2.91 during winter confirming the studied location was contaminated (PLI > 1) (Ali et al., 2016). The EF values of $\mathrm{Cd}$ were highest reaching 7.92 on average, indicating a high degree of anthropogenic contamination by this metal mainly from industrial activities. The mean values of $\mathrm{Zn}, \mathrm{Pb}, \mathrm{Cu}, \mathrm{Cr}$ and $\mathrm{Ni}$ were 2.25, 2.06, 2.04, 2.02 and 1.95, respectively indicating that they also originated from anthropogenic sources in most samples (Tang et al., 2013). Co and $\mathrm{Pb}$ varied from no enrichment to minor enrichment; $\mathrm{Ni}$ and $\mathrm{Zn}$ displayed no enrichment to moderate; no enrichment to moderately severe was shown by $\mathrm{Cr}$ and $\mathrm{Cu}$, while Cd displayed EF of moderate to moderately severe and minor to severe, respectively (Ekengele et al., 2017).

Therefore, the present study aims at identifying the sources of water pollution of El-Mariouteya canal (El-Moheet drain) and El-Rahawy drain, environmental risk assessment for soil, cultivated plants through using mathematical equations. 
J. Environ. Sci.

Institute of Environmental Studies and Research - Ain Shams University

\section{MATERIALS AND METHODS}

1. The study area: El- Mariouteya canal (El-Moheet drain) in El-Giza Governorate is considered one of the most polluted main drains, coming second to Bahr El-Baqar drain in the Eastern Delta Fig. (1a). It extended from the south Giza to north El-Riah El-Naseri.

2. Water, soils and plants sampling: Samples of water, (surface and subsurface soils) and plants are collected. Seventeen surface water samples, seven surface and sub-surface soil and twelve different plants. The samples were collected twice during summer and winter seasons, respectively. Sites along (ElMoheet and El-Rahawy drains) are selected Fig. (1b). Surface water samples were collected from different sites according to (EPA, 2007 and RMRS, 2012). Samples were brought to the lab in ice tank and stored at $4^{\circ} \mathrm{C}$ until analysis. Soil samples from agricultural areas are collected using an auger.

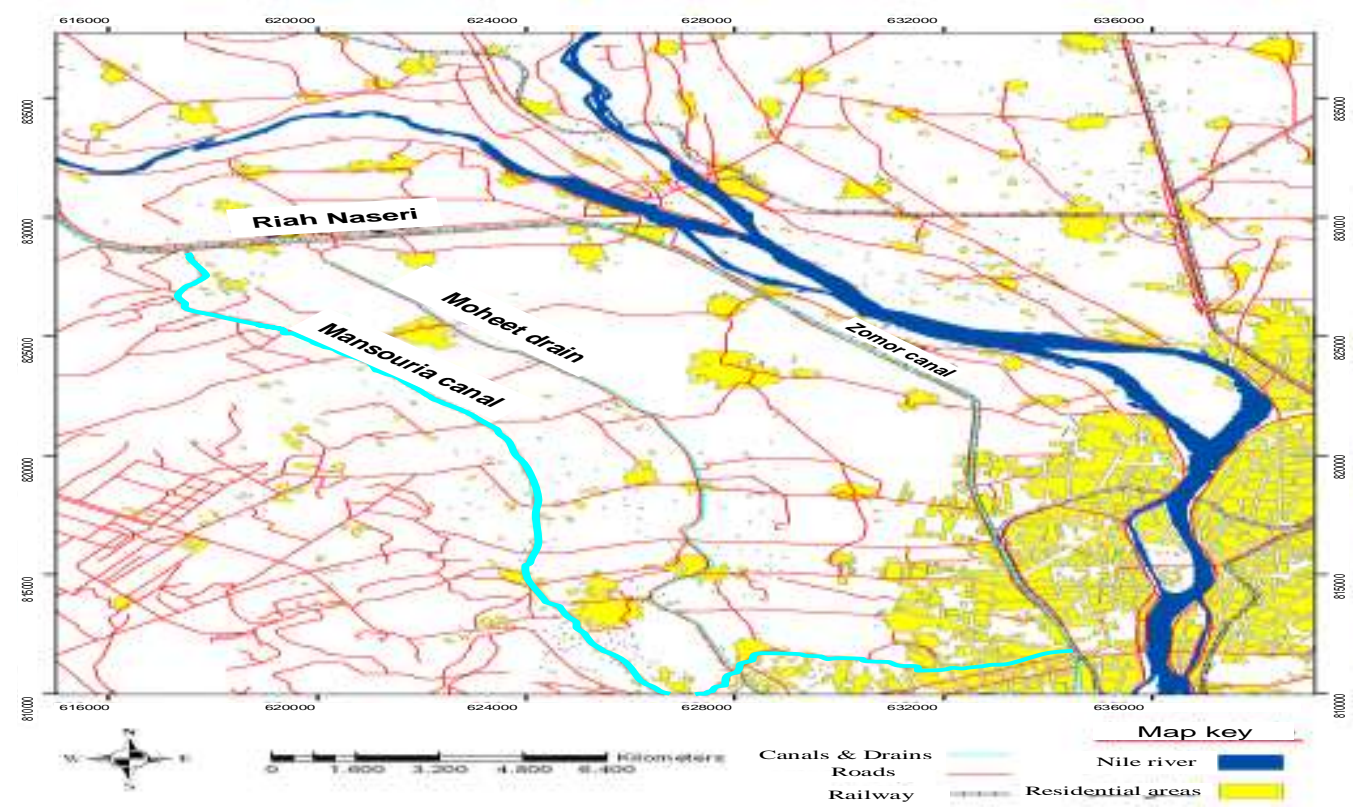

Fig. (1a): Location of the study area (El-Moheet drain). 


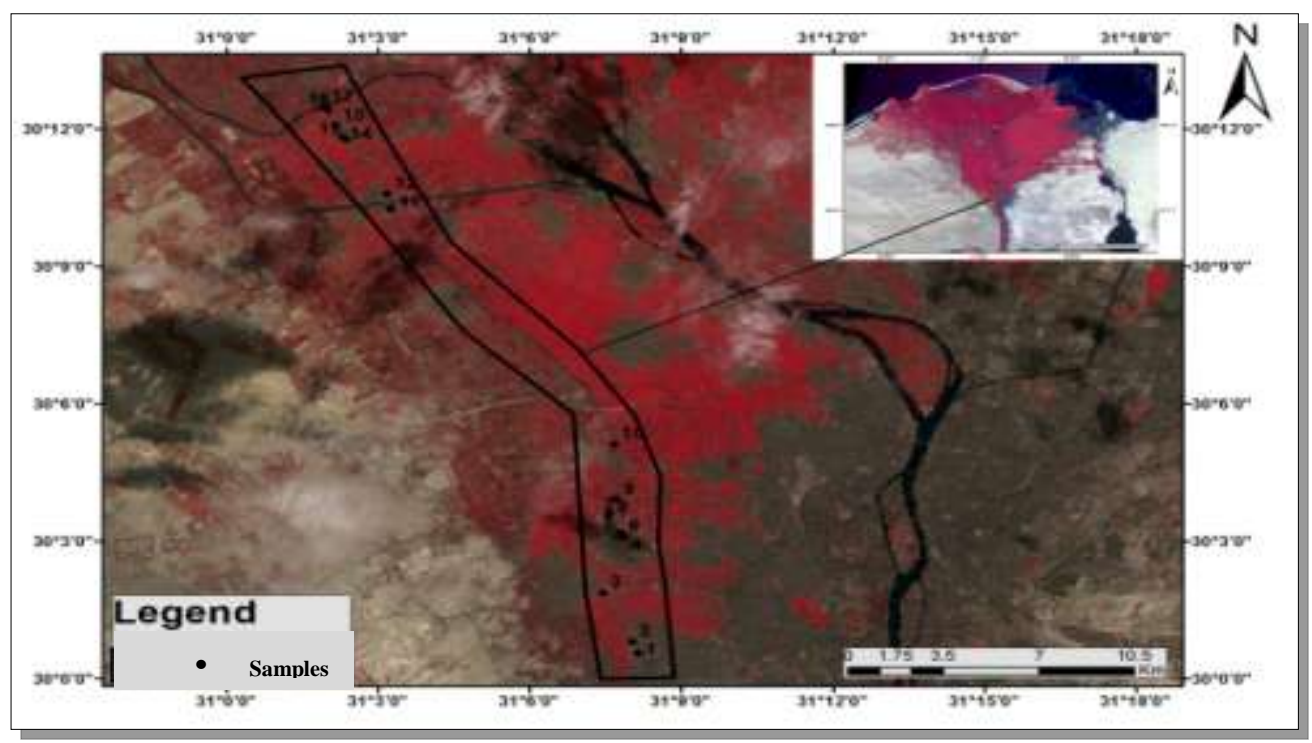

Fig. (1b): Map of the study area and sampling sites.

Soil samples were dried in air and crushed then sieved through a $<0.2$ mm sieve and stored in the labeled polythene sampling bags (Lei et al., 2008; Adepetu et al., 1996). A diversity of cereal crops and vegetables grown in the study area; Arugula (Eruca sativa), Okra fruits (Abelmoschus esculentus L. Moenth), Elephant grass (Pennisetum purpureum), Eggplant fruits (Solanum Melongena), Maize (Zea mays, L), Molokhia (Corchorus olitorius) were taken in summer. As well as Cabbage (Brassica oleracea var. capitata), Parsley (Petroselinum crispum), Wheat (Triticum aestivum), Mallow (Malva parviflora), Onion (Allium cepa) and Spinach (Spinacia oleracea) were taken in winter season. Different plants are collected from different sites of the sampling zone in 3-5 replicates and stored in labeled polythene sampling bags and brought to the lab, finally washed with tap water to remove any kind of contamination like soil particles then placed in drying oven for 72 hours at 6 
$70{ }^{\circ} \mathrm{C}$ after the dryness of the sample, it was completely grinded by using the stainless mill and digestion according to Adepetu et al., (1996).

3. Methods of analysis: Electrical conductivity (EC) in water and soil samples were determined by Electrical conductivity meter model WTW Series Cond 720; $\mathrm{pH}$ values in water, and soil suspensions (1:2.5) are determined by using pH meter model WTW Series $\mathrm{pH} 720$; as well as cations and anions in water and soil are determined according to ICARDA (2013). Available Fe, $\mathrm{Mn}, \mathrm{Zn}, \mathrm{Cu}, \mathrm{B} \mathrm{Cd}, \mathrm{Cr}, \mathrm{Co}, \mathrm{Ni}$ and $\mathrm{Pb}$ are extracted according to AB-DTPA (Soltanpour and Schwab, 1991). For total elements, samples (water, soil and plants) were digested by aqua regia according to (Cottenie et al., 1982; ICARDA. 2013). Different micro and heavy metals in water, soils and plants are determined according to (EPA, 1991) using Inductively Coupled Plasma (ICP) Spectrometry (Ultima 2 JY Plasma).

\section{Data analysis:}

4.1. Contamination Factors $(\mathbf{C F})$ : The contamination factor $(\mathrm{CF})$ is used to determine contamination status in the studied surface soil samples (Liu et al., 2005). The contamination factor is calculated according to Equation No. (1).

$$
C F=\frac{\text { Measured concentration }}{\text { Background concentration }}
$$

The background concentrations of different elements under study in $\mathrm{mg}$ $\mathrm{kg}^{-1}$ in the Earth's crust were 37.001 for Fe; 646 for Mn; 149 for Zn; 28.7 for $\mathrm{Cu} ; 18.98$ for $\mathrm{B} ; 14.9$ for $\mathrm{Co}$; 0.36 for $\mathrm{Cd} ; 122$ for $\mathrm{Cr} ; 57$ for $\mathrm{Ni}$ and 32.9 for $\mathrm{Pb} \mathrm{mgkg}^{-1}$ according to (Turekian and Wedepohl. 1961; Bradford et al., 1996). The significance of contamination factor and the level of contamination values are described according to Hakanson (1980). 
4.2. Contamination Degree $(\mathbf{C d})$ : The $\mathrm{Cd}$ is the sum of the contamination factors of all the elements examined according to Hakanson (1980).

$$
\mathrm{Cd}=\sum_{l=1}^{i=n} C F \quad \text { Equation No.(2). }
$$

\subsection{Modified degree of contamination $(\mathrm{mCd})$ :}

The (mCd) was defined as the sum of all contamination factors Abrahim (2005) and calculated as follows:

Where: $(\mathrm{n})=$ number ${ }^{\mathrm{mCd}}=\sum_{\mathrm{i}=1}^{\mathrm{p}=\mathrm{n}} C F / n \quad$ Equation No.(3) of analyzed elements; $(i=1)=$ the elements and $(\mathrm{CF})=$ contamination factor The classification and description of the modified degree of contamination $(\mathrm{mCd})$ in soil show the following gradations are proposed by Abrahim and Parker (2008) as shown in Table (1). CF and (Cd) were defined according to four categories as follows:-

Table (1): Contamination factor, degree of contamination level and modified degree of contamination.

\begin{tabular}{|c|c|c|c||}
\hline CF classes & Cd classes & mCd classes & Categories \\
\hline \hline- & - & $\mathrm{mCd}<1.5$ & Nil to very low contamination \\
\hline $\mathrm{CF}<1$ & $\mathrm{Cd}<9$ & $1.5 \leq \mathrm{mCd}<2$ & Low contamination \\
\hline $1 \leq \mathrm{CF}<3$ & $9 \leq \mathrm{Cd}<18$ & $2 \leq \mathrm{mCd}<4$ & Moderate contamination \\
\hline $3 \leq \mathrm{CF}<6$ & $18 \leq \mathrm{Cd}<36$ & $4 \leq \mathrm{mCd}<8$ & High contamination or \\
\hline $\mathrm{CF}>6$ & $\mathrm{Cd} \geq 36$ & $8 \leq \mathrm{mCd}<16$ & Very high contamination \\
\hline- & - & $16 \leq \mathrm{mCd}<32$ & Extremely high contamination \\
\hline- & - & $\mathrm{mCd} \geq 32$ & Ultra-high contamination \\
\hline
\end{tabular}

\subsection{The pollution load index (PLI):}

The PLI proposed by Tomlinson et al., (1980) is calculated using the following equation and level index tabulated in Table (2).

$$
\mathrm{PLI}=(\mathrm{CF} 1 \times \mathrm{CF} 2 \times \mathrm{CF} 3 \times \ldots . . \times \mathrm{CFn})^{1 / n} \quad \text { Equation No. (4) }
$$


J. Environ. Sci.

Institute of Environmental Studies and Research - Ain Shams University

Table (2): Pollution level index.

\begin{tabular}{|c|c||}
\hline PLI classes & Degree of pollution level \\
\hline \hline PLI $<1$ & Perfection \\
\hline PLI $=1$ & Base line Pollution level of pollution level index \\
\hline PLI $>1$ & Deterioration of site quality \\
\hline
\end{tabular}

4.5. Bio concentration factor (BCF): The $\mathrm{BCF}$ is calculated according to Liu et al., (2006) using the following equation

$$
\mathbf{B C F}=\mathbf{C}_{\text {plant }} / \mathbf{C}_{\text {soil }} \quad \text { Equation No. (5) }
$$

where:- $\mathrm{C}_{\text {plant }}$ is the concentration of elements in the plant and $\mathrm{C}$ soil is the concentration of the same elements in the soil on dry weight basis BCF $>1$ then the plants can be accumulators; $\mathrm{BCF}=1$ is no influences and $\mathrm{BCF}<1$ then the plant can be an excluder.

\section{RESULTS AND DISCUSSION}

\section{Assessment of irrigation water for EL-Mariouteya canal at different} sites during summer and winter seasons: Values of $\mathrm{pH}$; electrical conductivity $\left(\mathrm{EC} \mathrm{dSm}^{-1}\right)$; sodium absorption ratio (SAR) and residual sodium carbonate (RSC) along El-Mariouteya canal are listed in Table (3). Data revealed that the average of $\mathrm{pH}$ values ranged from 6.10 to 8.49 in summer season and 7.71 to 8.86 in winter season, respectively. EC values for irrigation water in summer season varied from 0.49 to $9.24 \mathrm{dSm}^{-1}$ and in winter season were 0.37 to $3.80 \mathrm{dSm}^{-1}$. SAR values ranged from (1.02 to 7.57) and (0.42 to 4.49) in summer and winter seasons, respectively. Samples are free from RSC in two seasons. Balkhair and Ashraf (2016) reported that the $\mathrm{pH}$ of irrigation water is not an acceptable criterion of water quality because it tends to be buffered by the soil and most crops can tolerate a wide $\mathrm{pH}$ range. It is clear from these results that the 
Taha, Aya, et al

values of $\mathrm{EC}$ in summer exceeded the degree of restriction on use (severe restriction). El Tohamy et al., (2015) reported that EC in El-Mariouteya Canal ranged between $\left(0.53\right.$ to $\left.5.26 \mathrm{dSm}^{-1}\right)$ and $\left(1.26\right.$ to $\left.6.72 \mathrm{dSm}^{-1}\right)$ in winter and summer seasons, respectively. On the other hand, EC indicates a slight to moderate degree of restriction on the use of this water for irrigation in winter season according to the standards of the Food and Agriculture Organization (FAO) of the United Nations, (Ayers and Westcot, 1985). Therefore, it is necessary to control the salinity when using this wastewater for irrigation purposes.

Table (3): Assessment of chemical analysis in irrigation water for ElMariouteya canal.

\begin{tabular}{|c|c|c|c|c|c|c|}
\hline Sites No. & pH & $\begin{array}{c}\text { EC } \\
\text { dS m }\end{array}$ & SAR & pH & $\begin{array}{c}\text { EC } \\
\mathbf{d S ~ m}^{-1}\end{array}$ & SAR \\
\hline & \multicolumn{3}{|c|}{ Summer season } & \multicolumn{3}{c|}{ Winter season } \\
\hline \hline 1 & 6.10 & 6.51 & 6.91 & 8.77 & 2.51 & 3.07 \\
\hline 2 & 8.46 & 7.95 & 7.57 & 8.00 & 3.80 & 4.49 \\
\hline 3 & 8.49 & 7.70 & 7.52 & 8.60 & 2.73 & 3.40 \\
\hline 4 & 8.28 & 7.80 & 7.53 & 8.86 & 2.23 & 3.07 \\
\hline 5 & 8.06 & 2.04 & 3.88 & 8.67 & 2.01 & 1.75 \\
\hline 6 & 7.95 & 8.66 & 6.61 & 8.80 & 2.22 & 3.18 \\
\hline 7 & 7.76 & 8.81 & 3.68 & 8.64 & 1.20 & 1.19 \\
\hline 8 & 7.71 & 9.24 & 5.96 & 8.69 & 1.46 & 2.02 \\
\hline 9 & 8.15 & 6.07 & 6.67 & 8.35 & 1.48 & 2.02 \\
\hline 10 & 8.30 & 5.91 & 6.81 & 8.71 & 1.39 & 1.63 \\
\hline 11 & 7.79 & 1.99 & 3.76 & 8.36 & 0.99 & 1.20 \\
\hline 12 & 7.60 & 2.01 & 3.67 & 8.28 & 1.00 & 1.10 \\
\hline 13 & 7.57 & 2.13 & 3.23 & 8.51 & 0.81 & 0.42 \\
\hline 14 & 7.57 & 1.98 & 3.61 & 8.53 & 0.98 & 0.72 \\
\hline 15 & 7.39 & 1.85 & 3.35 & 8.34 & 0.95 & 0.87 \\
\hline 16 & 7.23 & 1.97 & 2.89 & 8.37 & 1.10 & 1.14 \\
\hline 17 & 7.48 & 0.49 & 1.02 & 7.71 & 0.37 & 0.64 \\
\hline Min. & 6.10 & 0.49 & 1.02 & 7.71 & 0.37 & 0.42 \\
\hline Max. & 8.49 & 9.24 & 7.57 & 8.86 & 3.80 & 4.49 \\
\hline
\end{tabular}

- RSC is zero in summer and winter seasons. 
2. Micro-nutrients and heavy metals in irrigation water: The results of Tables (4 and 5) revealed that the concentrations $\left(\mathrm{mg}^{-1}\right)$ of micro-nutrients and heavy metals in El-Mariouteya canal water during summer and winter seasons. Also, results indicated that the mean concentrations $\left(\mathrm{mg}^{-1}\right.$ ) of elements in water were highest relatively for the Fe followed by $\mathrm{Zn}, \mathrm{Mn}, \mathrm{B}$, $\mathrm{Pb}, \mathrm{Cu}, \mathrm{Cr}, \mathrm{Ni}, \mathrm{Co}$ and $\mathrm{Cd}$.

Data showed that the mean heavy metal concentrations displayed the following decreasing order: $\mathrm{Zn}>\mathrm{Mn}>\mathrm{Fe}>\mathrm{B}>\mathrm{Pb}>\mathrm{Ni}>\mathrm{Co} \approx \mathrm{Cr}$. Regarding the concentration of $\mathrm{Cd}, \mathrm{Co}, \mathrm{Cr}, \mathrm{Ni}$ and $\mathrm{Pb}$ are very low compared with standard limits for irrigation water (FAO 1985 and 1992). As shown from Tables (4 and 5), the average micro-nutrients and heavy metal concentrations in summer season are higher than the average values in winter season. However, none of the heavy metals exceeded the recommended maximum concentrations of trace elements in irrigated water (Ayers and Westcot, 1985; FAO, 1992; Row and Abdel-Majid, 1995). This is mainly due to the clay texture of the sediment which can accumulate excessive amounts of elements on the surface. These results are in a good agreement with (ElKholy et al., 2015; Sherif et al., 2015) which reported that the values of trace elements are considerably below the permissible limits. 
Table (4): Assessment of micro-nutrients in irrigation water for ElMariouteya canal.

\begin{tabular}{|c|c|c|c|c|c|c|c|c|c|c|}
\hline \multirow{2}{*}{$\begin{array}{l}\text { Sites } \\
\text { No. }\end{array}$} & \multicolumn{10}{|c|}{ Concentration $\mathrm{mg} \mathrm{l}^{-1}$} \\
\hline & $\mathrm{Fe}$ & Mn & $\mathbf{Z n}$ & $\mathbf{C u}$ & B & Fe & Mn & $\mathbf{Z n}$ & $\mathrm{Cu}$ & B \\
\hline & \multicolumn{5}{|c|}{ Summer season } & \multicolumn{5}{|c|}{ Winter season } \\
\hline 1 & 0.416 & 0.155 & 0.863 & 0.080 & 0.130 & 0.080 & 0.092 & 0.025 & 0.066 & 0.060 \\
\hline 2 & 523 & 0.067 & 0.654 & 0.050 & 0.150 & 0.160 & 0.125 & 0.030 & 0.046 & \\
\hline 3 & 602 & 082 & 0.160 & 0.073 & 0.146 & 0.080 & 0.501 & 0.095 & 0.050 & \\
\hline 4 & & & 0 & 0.0 & & & 057 & 0.440 & 0.054 & \\
\hline 5 & & & 0.418 & 0.053 & 0.1 & & 071 & 0.420 & 0.030 & 095 \\
\hline 6 & 017 & 77 & 0.1 & 0.050 & 0.0 & & 0.554 & 0.362 & 0.038 & 0.086 \\
\hline 7 & 0.506 & 0.201 & 0.898 & 0.088 & 0.1 & 0.1 & 0.160 & 0.250 & 0.040 & 0.040 \\
\hline 8 & 794 & 0.136 & 0.147 & 0.091 & 0.128 & 0.090 & 0.246 & 0.360 & 0.030 & 0.060 \\
\hline 9 & 310 & & 0.468 & 0.086 & 0.457 & 0.090 & 0.082 & 0.480 & 0.040 & 0.070 \\
\hline 10 & 465 & & $0.4 \varepsilon$ & 0.0 & & & 0.060 & 0.250 & 0.027 & 0.102 \\
\hline 11 & 286 & 0.316 & 0.483 & 0.050 & 0.249 & 0.150 & 0.065 & 0.315 & 0.031 & 0.110 \\
\hline 12 & 1.925 & 0.179 & 1.320 & 0.040 & 0.206 & 0.130 & 0.120 & 0.286 & 0.023 & 0.113 \\
\hline 13 & 0.645 & 0.203 & 0.253 & 0.055 & 0.322 & 0.130 & 0.105 & 0.300 & 0.040 & 0.123 \\
\hline 14 & 311 & & 0.178 & 0.138 & 0.1 & 0.1 & 0.074 & 0.170 & 0.030 & 0.072 \\
\hline 15 & 325 & & 0.145 & 0.040 & 0.128 & 0.100 & 0.069 & 0.110 & 0.030 & 0.120 \\
\hline 16 & 263 & & 0.111 & 0.030 & 0.126 & 0.140 & 0.052 & 0.090 & 0.024 & 0.118 \\
\hline 17 & & & 0.088 & 0.004 & 0.032 & 0.050 & 0.016 & 0.030 & 0.003 & 0.002 \\
\hline Min. & 0.105 & 0.035 & 0.088 & 0.004 & 0.032 & 0.050 & 0.016 & 0.025 & 0.003 & 0.002 \\
\hline Max. & 2.465 & 0.677 & 4.830 & 0.138 & 0.457 & 0.380 & 0.554 & 0.480 & 0.066 & 0.149 \\
\hline Average & 0.789 & & 0.704 & 0.060 & \begin{tabular}{|l|l|}
0.177 \\
\end{tabular} & 0.131 & 0.144 & 0.236 & 0.035 & 0.094 \\
\hline$*$ & 5.00 & 0.20 & 2.00 & 0.020 & 0.70 & 5.00 & 0.20 & \begin{tabular}{|l|}
2.00 \\
\end{tabular} & 0.020 & 0.70 \\
\hline
\end{tabular}

*Permissible limit (Ayers and Westcot, 1985). 
J. Environ. Sci.

Institute of Environmental Studies and Research - Ain Shams University

Table (5): Assessment of heavy metals in irrigation water for El-Mariouteya canal.

\begin{tabular}{|c|c|c|c|c|c|c|c|c|c|c|}
\hline \multirow{2}{*}{$\begin{array}{c}\text { Sites } \\
\text { No. }\end{array}$} & \multicolumn{10}{|c|}{ Concentration $\mathrm{mg} \mathrm{l}^{-1}$} \\
\hline & Cd & Co & $\mathrm{Cr}$ & $\mathbf{N i}$ & $\mathbf{P b}$ & Cd & Co & $\mathrm{Cr}$ & $\mathbf{N i}$ & $\mathbf{P b}$ \\
\hline & \multicolumn{5}{|c|}{ Summer season } & \multicolumn{5}{|c|}{ Winter season } \\
\hline 1 & 000 & 0.001 & 0.003 & 0.003 & 0.0 & & 0.000 & 0.002 & 0.001 & 0.002 \\
\hline 2 & 000 & 001 & 003 & 0.0 & 0.006 & 000 & 0.000 & 0.002 & 0.00 & 0.002 \\
\hline 3 & 000 & 001 & 002 & & & & 0.000 & & & \\
\hline 4 & 000 & 1 & 001 & 0.0 & 0.0 & 00 & 0.000 & 0.000 & 0.0 & 0.009 \\
\hline 5 & 003 & 002 & 002 & 0.00 & 0.010 & 0.001 & 0.001 & 0.001 & 0.001 & 0.005 \\
\hline 6 & 000 & 3 & 2 & 0.0 & 0.0 & & & 0.0 & 0.0 & 0.012 \\
\hline 7 & 002 & 003 & 0.002 & 0.00 & 0.1 & 0.001 & 0.002 & 0.001 & 0.001 & 0.007 \\
\hline 8 & & & 2 & & & & 0.001 & 0.000 & & 0.014 \\
\hline 9 & 005 & 2 & 0.004 & 0.0 & 0.0 & 0.0 & 0.002 & 0.002 & 0.004 & 0.006 \\
\hline 10 & 02 & 2 & 26 & 0.0 & 0. & & 0.000 & 0.001 & 0.003 & 0.014 \\
\hline 11 & 000 & 2 & 4 & & 0.0 & & 0.000 & 0.003 & 0.005 & 0.002 \\
\hline 12 & 000 & 0.003 & 0.003 & 0.006 & 0.016 & 0.000 & 0.002 & 0.002 & 0.002 & 0.012 \\
\hline 13 & 004 & 001 & 0.023 & 0.0 & 0.0 & & 0.002 & 0.002 & 0.001 & 0.012 \\
\hline 14 & 002 & 0.003 & 0.010 & 0.009 & 0.019 & 0.001 & 0.000 & 0.003 & 0.002 & 0.006 \\
\hline 15 & 000 & 002 & 0.01 & 0.0 & 0.0 & 0.0 & 0.000 & 0.001 & 0.002 & 0.010 \\
\hline 16 & 000 & 000 & 0.002 & 0.00 & 0.010 & 0.000 & 0.000 & 0.001 & 0.001 & 0.002 \\
\hline 17 & 000 & 0.000 & 0.000 & 0.001 & 0.007 & 0.000 & 0.000 & 0.000 & 0.000 & 0.000 \\
\hline Min. & 000 & 0.000 & 0.000 & 0.001 & 0.006 & 0.000 & 0.000 & 0.000 & 0.000 & 0.000 \\
\hline Max. & 0.005 & 0.003 & 0.026 & 0.009 & 0.110 & 0.002 & 0.002 & 0.003 & 0.005 & 0.014 \\
\hline Average & 0.001 & 0.002 & 0.006 & 0.004 & 0.025 & 0.000 & 0.001 & 0.001 & 0.002 & 0.007 \\
\hline * & 0.01 & -- & 0.10 & 0.20 & 5.00 & \begin{tabular}{|l|}
0.01 \\
\end{tabular} & -- & 0.10 & 0.20 & 5.00 \\
\hline
\end{tabular}

*Permissible limit (Ayers and Westcot, 1985).

\section{3. pH and salinity in soils irrigated from El-Mariouteya canal at different} sites during summer and winter seasons:

Soil $\mathrm{pH}$ values in different sites are listed in Table (6). The $\mathrm{pH}$ values for sites tend to be normal and slightly alkaline in all sites. Fatih et al., (2007) 
found that soil $\mathrm{pH}$ values increased with soil depth, while soil irrigated with wastewater were lower compared to soil irrigated with non-wastewater; this was probably ascribed to high load of organic matter in wastewater. Results in Table (6) showed that the soil $\mathrm{pH}$ and EC values ranged from (7.18 to 7.98 and 7.56 to 7.93 ) and (2.04 to 17.50 and 1.36 to $7.70 \mathrm{dS} \mathrm{m}^{-1}$ ) in summer and winter seasons, respectively.

Table (6): Assessment of chemical analysis in surface and sub-surface soil irrigated from El-Mariouteya canal at different sites during summer and winter seasons.

\begin{tabular}{|c|c|c|c|c|c|}
\hline \multirow[t]{2}{*}{ Sites No. } & \multirow{2}{*}{$\begin{array}{l}\text { Soil depth in } \\
\text { cm }\end{array}$} & pH & $\begin{array}{c}\text { EC } \\
\text { dS m }^{-1}\end{array}$ & $\mathbf{p H}$ & $\begin{array}{c}E C \\
d_{S} ~ m^{-1} \\
\end{array}$ \\
\hline & & \multicolumn{2}{|c|}{ Summer season } & \multicolumn{2}{|c|}{ Winter season } \\
\hline \multirow{2}{*}{1} & $0-20$ & 7.30 & 11.62 & 7.80 & 7.70 \\
\hline & $20-40$ & 7.84 & 4.19 & 7.90 & 4.77 \\
\hline \multirow{2}{*}{2} & $0-20$ & 7.40 & 13.18 & 7.80 & 3.55 \\
\hline & $20-40$ & 7.60 & 4.12 & 7.93 & 3.43 \\
\hline \multirow{2}{*}{3} & $0-20$ & 7.18 & 4.95 & 7.90 & 4.15 \\
\hline & $20-40$ & 7.98 & 3.26 & 7.90 & 3.09 \\
\hline \multirow{2}{*}{8} & $0-20$ & 7.70 & 17.50 & 7.66 & 6.84 \\
\hline & $20-40$ & 7.70 & 10.80 & 7.66 & 2.09 \\
\hline \multirow{2}{*}{10} & $0-20$ & 7.70 & 15.25 & 7.72 & 5.75 \\
\hline & $20-40$ & 7.60 & 6.85 & 7.72 & 2.49 \\
\hline \multirow{2}{*}{11} & $0-20$ & 7.55 & 17.40 & 7.56 & 4.89 \\
\hline & $20-40$ & 7.90 & 2.97 & 7.80 & 2.90 \\
\hline \multirow{2}{*}{15} & $0-20$ & 7.60 & 5.99 & 7.75 & 3.55 \\
\hline & $20-40$ & 7.90 & 2.04 & 7.69 & 1.36 \\
\hline \multicolumn{2}{|c|}{ Minimum } & 7.18 & 2.04 & 7.56 & 1.36 \\
\hline \multicolumn{2}{|c|}{ Maximum } & 7.98 & 17.50 & 7.93 & 7.70 \\
\hline \multicolumn{2}{|c|}{ Average } & 7.64 & 8.58 & 7.77 & 4.04 \\
\hline
\end{tabular}

Increasing in soil salinity may be due to high concentration of salts in agricultural drainage water used in irrigation as well as the untreated domestic 14

Vol. 46, No.2, Jun. 2019 
wastewater and human activities which discharge along El-Moheet and ElRahawy drains. High temperature in summer season leads to high evaporation from soil surface. Using this type of water which contains high salts and evaporation water from soil surface leading to drying then rise of salts by the poetic property to surface soil, leading to the increase of concentration of salts in the soil.

4. Available micro-nutrients and heavy metal contents in soils: Available concentrations of micro-nutrient and heavy metals in surface and sub-surface soils irrigated from El-Mariouteya canal at different sites during summer and winter seasons are presented in Tables (7 and 8). Data explained that available $\mathrm{Fe}, \mathrm{Mn}, \mathrm{Zn}$ and $\mathrm{Cu}$ in summer and winter season are very high concentration more highly limits allowed according to Soltanpour and Schwab (1991), but $\mathrm{B}, \mathrm{Cd}, \mathrm{Co}, \mathrm{Cr}, \mathrm{Ni}$ and $\mathrm{Pb}$ are within the safe limits allowed according to (Soltanpour and Schwab. 1991; Elrashidi et al., 2003; Michael et al., 2007). The averages of available elements of soils in summer and winter seasons were $(23.335,7.743,4.319,6.576,0.084,0.017,0.050$, $0.018,0.375$ and 1.129) and $(17.512,6.230,3.282,5.508,0.068,0.01,0.035$, 0.013, 0.307 and 0.830) for $\mathrm{Fe}, \mathrm{Mn}, \mathrm{Zn}, \mathrm{Cu}, \mathrm{B}, \mathrm{Cd}, \mathrm{Co}, \mathrm{Cr}, \mathrm{Ni}$ and $\mathrm{Pb}$, respectively. 
Table (7): Assessment of available micro nutrients in surface and sub-surface soil irrigated from El-Mariouteya canal.

\begin{tabular}{|c|c|c|c|c|c|c|c|c|c|c|c|}
\hline \multirow{3}{*}{ Sites No. } & \multirow{3}{*}{$\begin{array}{c}\text { Soil depth } \\
\quad(\mathrm{cm})\end{array}$} & \multicolumn{10}{|c|}{ Concentration $\mathrm{mg} \mathrm{kg}^{-1}$} \\
\hline & & $\mathrm{Fe}$ & $\mathrm{Mn}$ & $\mathrm{Zn}$ & $\mathrm{Cu}$ & $\mathrm{B}$ & $\mathrm{Fe}$ & $\mathrm{Mn}$ & $\mathrm{Zn}$ & $\mathrm{Cu}$ & $\mathrm{B}$ \\
\hline & & \multicolumn{5}{|c|}{ Summer season } & \multicolumn{5}{|c|}{ Winter season } \\
\hline \multirow{2}{*}{1} & $0-20$ & 24.268 & 7.652 & 3.758 & 6.636 & 0.100 & 17.454 & 6.214 & 3.362 & 6.144 & 0.090 \\
\hline & $20-40$ & 21.220 & 5.586 & 3.052 & 5.868 & 0.044 & 15.076 & 5.966 & 3.220 & 5.348 & 0.038 \\
\hline \multirow{2}{*}{2} & $0-20$ & 12.814 & 12.454 & 3.704 & 4.784 & 0.180 & 9.620 & 8.866 & 3.316 & 4.784 & 0.128 \\
\hline & $20-40$ & 12.078 & 8.130 & 2.092 & 4.600 & 0.052 & 8.468 & 5.806 & 1.824 & 4.378 & 0.058 \\
\hline \multirow{2}{*}{3} & $0-20$ & 18.520 & 7.054 & 3.524 & 6.192 & 0.220 & 16.078 & 8.106 & 2.876 & 4.726 & 0.204 \\
\hline & $20-40$ & 14.384 & 2.760 & 2.800 & 6.012 & 0.082 & 11.170 & 4.520 & 2.094 & 4.418 & 0.062 \\
\hline \multirow{2}{*}{8} & $0-20$ & 24.598 & 10.630 & 4.984 & 5.858 & 0.080 & 9.968 & 10.190 & 3.862 & 5.324 & 0.082 \\
\hline & $20-40$ & 19.116 & 8.144 & 2.692 & 5.646 & 0.040 & 4.510 & 4.424 & 1.872 & 5.324 & 0.034 \\
\hline \multirow{2}{*}{10} & $0-20$ & 52.910 & 8.516 & 9.502 & 12.382 & 0.116 & 47.366 & 8.128 & 8.463 & 9.712 & 0.056 \\
\hline & $20-40$ & 42.408 & 7.596 & 7.428 & 9.398 & 0.040 & 39.190 & 5.718 & 7.486 & 8.064 & 0.038 \\
\hline \multirow{2}{*}{11} & $0-20$ & 31.614 & 11.076 & 8.172 & 7.472 & 0.110 & 20.170 & 7.756 & 3.214 & 6.614 & 0.084 \\
\hline & $20-40$ & 21.218 & 5.322 & 4.908 & 7.344 & 0.044 & 15.570 & 4.128 & 1.662 & 5.380 & 0.036 \\
\hline \multirow{2}{*}{15} & $0-20$ & 16.724 & 7.648 & 2.350 & 5.032 & 0.044 & 15.910 & 4.066 & 1.854 & 3.448 & 0.028 \\
\hline & $20-40$ & 14.814 & 5.828 & 1.506 & 4.838 & 0.024 & 14.622 & 3.332 & 0.848 & 3.442 & 0.020 \\
\hline \multicolumn{2}{|c|}{ Minimum } & 12.078 & 2.760 & 1.506 & 4.600 & 0.024 & 4.510 & 3.332 & 0.848 & 3.442 & 0.020 \\
\hline \multicolumn{2}{|c|}{ Maximum } & 52.910 & 12.454 & 9.502 & 12.382 & 0.220 & 47.366 & 10.190 & 8.463 & 9.712 & 0.204 \\
\hline \multicolumn{2}{|c|}{ Average } & 23.335 & 7.743 & 4.319 & 6.576 & 0.084 & 17.512 & 6.230 & 3.282 & 5.508 & 0.068 \\
\hline \multicolumn{2}{|c|}{ Critical limit } & $>5.0^{\mathrm{a}}$ & $>1.0^{\mathrm{a}}$ & $>1.5^{\mathrm{a}}$ & $>0.5^{\mathrm{a}}$ & $0.80^{\mathrm{b}}$ & $>5.0^{\mathrm{a}}$ & $>1.0^{\mathrm{a}}$ & $>1.5^{\mathrm{a}}$ & $>0.5^{\mathrm{a}}$ & $0.80^{\mathrm{b}}$ \\
\hline
\end{tabular}

a- Soltanpour and Schwab (1991), b- Michael et al., (2007). 
J. Environ. Sci.

Institute of Environmental Studies and Research - Ain Shams University

Table (8): Assessment of available heavy metals in surface and sub-surface soil irrigated from El-Mariouteya canal.

\begin{tabular}{|c|c|c|c|c|c|c|c|c|c|c|c|}
\hline \multirow{3}{*}{ Sites No. } & \multirow{3}{*}{$\begin{array}{c}\text { Soil depth } \\
(\mathrm{cm})\end{array}$} & \multicolumn{10}{|c|}{ Concentration $\mathrm{mg} \mathrm{kg}^{-1}$} \\
\hline & & $\mathrm{Cd}$ & $\mathrm{Co}$ & $\mathrm{Cr}$ & $\mathrm{Ni}$ & $\mathrm{Pb}$ & $\mathrm{Cd}$ & $\mathrm{Co}$ & $\mathrm{Cr}$ & $\mathrm{Ni}$ & $\mathrm{Pb}$ \\
\hline & & \multicolumn{5}{|c|}{ Summer season } & \multicolumn{5}{|c|}{ Winter season } \\
\hline \multirow{2}{*}{1} & $0-20$ & 0.016 & 0.038 & 0.020 & 0.290 & 1.748 & 0.016 & 0.026 & 0.014 & 0.252 & 1.204 \\
\hline & $20-40$ & 0.012 & 0.022 & 0.012 & 0.276 & 1.660 & 0.012 & 0.012 & 0.012 & 0.194 & 0.832 \\
\hline \multirow{2}{*}{2} & $0-20$ & 0.028 & 0.114 & 0.018 & 0.546 & 1.642 & 0.010 & 0.086 & 0.010 & 0.342 & 1.414 \\
\hline & $20-40$ & 0.008 & 0.090 & 0.006 & 0.390 & 1.194 & 0.008 & 0.062 & 0.004 & 0.168 & 1.190 \\
\hline \multirow{2}{*}{3} & $0-20$ & 0.016 & 0.060 & 0.024 & 0.368 & 0.918 & 0.010 & 0.046 & 0.020 & 0.344 & 0.594 \\
\hline & $20-40$ & 0.012 & 0.012 & 0.010 & 0.332 & 0.624 & 0.006 & 0.004 & 0.004 & 0.284 & 0.242 \\
\hline \multirow{2}{*}{8} & $0-20$ & 0.024 & 0.064 & 0.048 & 0.426 & 1.476 & 0.008 & 0.038 & 0.036 & 0.396 & 0.724 \\
\hline & $20-40$ & 0.022 & 0.054 & 0.016 & 0.378 & 1.268 & 0.008 & 0.014 & 0.008 & 0.230 & 0.586 \\
\hline \multirow{2}{*}{10} & $0-20$ & 0.024 & 0.062 & 0.018 & 0.404 & 1.358 & 0.018 & 0.052 & 0.016 & 0.654 & 1.486 \\
\hline & $20-40$ & 0.018 & 0.054 & 0.016 & 0.378 & 1.176 & 0.012 & 0.052 & 0.012 & 0.442 & 1.110 \\
\hline \multirow{2}{*}{11} & $0-20$ & 0.020 & 0.070 & 0.026 & 0.764 & 1.258 & 0.012 & 0.044 & 0.020 & 0.354 & 0.974 \\
\hline & $20-40$ & 0.020 & 0.030 & 0.018 & 0.260 & 0.704 & 0.010 & 0.020 & 0.014 & 0.162 & 0.690 \\
\hline \multirow{2}{*}{15} & $0-20$ & 0.012 & 0.020 & 0.008 & 0.236 & 0.416 & 0.006 & 0.020 & 0.006 & 0.286 & 0.316 \\
\hline & $20-40$ & 0.012 & 0.016 & 0.006 & 0.196 & 0.358 & 0.006 & 0.014 & 0.006 & 0.190 & 0.264 \\
\hline \multicolumn{2}{|c|}{ Minimum } & 0.008 & 0.012 & 0.006 & 0.196 & 0.358 & 0.006 & 0.004 & 0.004 & 0.162 & 0.242 \\
\hline \multicolumn{2}{|c|}{ Maximum } & 0.028 & 0.114 & 0.048 & 0.764 & 1.748 & 0.018 & 0.086 & 0.036 & 0.654 & 1.486 \\
\hline \multicolumn{2}{|c|}{ Average } & 0.017 & 0.050 & 0.018 & 0.375 & 1.129 & 0.010 & 0.035 & 0.013 & 0.307 & 0.830 \\
\hline \multicolumn{2}{|c|}{ Critical limit } & $0.31^{b}$ & $--b$ & $8.0^{b}$ & $8.1^{\mathrm{b}}$ & $13^{b}$ & $0.31^{\mathrm{b}}$ & $--\mathrm{b}$ & $8.0^{b}$ & $8.1^{b}$ & $13^{b}$ \\
\hline
\end{tabular}

b- Michael et al., (2007).

5. Total micro-nutrients and heavy metal contents in soils: The values of micro-nutrients and heavy metals contents are presented in Tables (9 and 10). The sequence of heavy metals according to their total concentrations in surface and sub-surface soil during summer season was $\mathrm{Fe}>\mathrm{Mn}>\mathrm{Cr}>\mathrm{Zn}>$ $\mathrm{Cu}>\mathrm{Ni}>\mathrm{Co}>\mathrm{Pb}>\mathrm{Cd}>\mathrm{B}$. The sequence of micro elements and heavy metals according to their total concentrations in surface and subsurface soil during winter season was $\mathrm{Fe}>\mathrm{Mn}>\mathrm{Cr}>\mathrm{Zn}>\mathrm{Cu}>\mathrm{Ni}>\mathrm{Co}>\mathrm{Pb}>\mathrm{Cd}>\mathrm{B}$. 
Taha, Aya, et al

Table (9): Assessment of total micro nutrients and heavy metals in surface and sub-surface soil irrigated from El-Mariouteya canal.

\begin{tabular}{|c|c|c|c|c|c|c|c|c|c|c|c|}
\hline \multirow{3}{*}{ Sites No. } & \multirow{3}{*}{$\begin{array}{c}\text { Soil } \\
\text { depth } \\
(\mathrm{cm})\end{array}$} & \multicolumn{10}{|c|}{ Concentration mg kg-1 } \\
\hline & & $\mathrm{Fe}$ & $\mathrm{Mn}$ & $\mathrm{Zn}$ & $\mathrm{Cu}$ & $\mathrm{B}$ & $\mathrm{Fe}$ & $\mathrm{Mn}$ & $\mathrm{Zn}$ & $\mathrm{Cu}$ & $\mathrm{B}$ \\
\hline & & \multicolumn{5}{|c|}{ Summer season } & \multicolumn{5}{|c|}{ Winter season } \\
\hline \multirow{2}{*}{1} & $0-20$ & 73410 & 884.7 & 73.0 & 52.9 & 4.1 & 70470 & 705.2 & 67.3 & 51.7 & 3.9 \\
\hline & $20-40$ & 67920 & 642.0 & 66.6 & 50.8 & 3.5 & 65655 & 641.2 & 51.8 & 50.9 & 3.4 \\
\hline \multirow{2}{*}{2} & $0-20$ & 30899 & 643.5 & 67.5 & 48.2 & 2.6 & 27466 & 597.8 & 62.8 & 47.3 & 2.2 \\
\hline & $20-40$ & 27299 & 545.5 & 53.0 & 42.5 & 2.2 & 25023 & 590.1 & 52.5 & 41.9 & 2.1 \\
\hline \multirow{2}{*}{3} & $0-20$ & 58620 & 875.7 & 74.6 & 68.6 & 3.6 & 53591 & 734.0 & 61.2 & 52.0 & 2.9 \\
\hline & $20-40$ & 58410 & 832.2 & 72.8 & 53.3 & 3.6 & 52591 & 733.0 & 57.3 & 48.2 & 2.3 \\
\hline \multirow{2}{*}{8} & $0-20$ & 35775 & 773.5 & 69.5 & 55.0 & 2.6 & 34073 & 722.4 & 67.1 & 44.4 & 2.1 \\
\hline & $20-40$ & 32600 & 534.6 & 49.0 & 55.0 & 2.5 & 32382 & 699.1 & 48.8 & 43.6 & 2.0 \\
\hline \multirow{2}{*}{10} & $0-20$ & 38831 & 818.6 & 90.4 & 68.0 & 2.7 & 36855 & 816.5 & 87.8 & 50.2 & 2.2 \\
\hline & $20-40$ & 36475 & 674.0 & 81.2 & 54.7 & 2.2 & 33767 & 695.7 & 83.0 & 49.4 & 1.8 \\
\hline \multirow{2}{*}{11} & $0-20$ & 34812 & 802.8 & 89.2 & 64.2 & 2.5 & 33333 & 582.2 & 64.1 & 43.3 & 1.6 \\
\hline & $20-40$ & 32664 & 642.9 & 73.3 & 50.3 & 2.2 & 31452 & 547.5 & 51.8 & 43.4 & 1.5 \\
\hline \multirow{2}{*}{15} & $0-20$ & 35335 & 968.6 & 75.2 & 46.3 & 1.4 & 33668 & 634.0 & 56.6 & 43.6 & 1.3 \\
\hline & $20-40$ & 32507 & 823.3 & 65.0 & 45.0 & 1.2 & 30276 & 551.9 & 53.4 & 43.5 & 1.3 \\
\hline \multicolumn{2}{|c|}{ Minimum } & 27299 & 534.6 & 49.0 & 42.5 & 1.2 & 25023 & 547.5 & 48.8 & 41.9 & 1.3 \\
\hline \multicolumn{2}{|c|}{ Maximum } & 58620 & 968.6 & 90.4 & 68.6 & 3.6 & 70470 & 816.5 & 87.8 & 52.0 & 3.9 \\
\hline \multicolumn{2}{|c|}{ Average } & 37852 & 744.6 & 71.7 & 54.3 & 2.4 & 40043 & 660.8 & 61.8 & 46.7 & 2.2 \\
\hline \multicolumn{2}{|c|}{ Critical limit } & $\begin{array}{c}200- \\
50000^{\mathrm{a}}\end{array}$ & $\begin{array}{c}20- \\
10000^{\mathrm{a}}\end{array}$ & $\begin{array}{l}300- \\
600^{b}\end{array}$ & $\begin{array}{c}2- \\
250^{\mathrm{a}}\end{array}$ & -- & $\begin{array}{c}200- \\
50000^{\mathrm{a}}\end{array}$ & $\begin{array}{c}20- \\
10000^{\mathrm{a}}\end{array}$ & $\begin{array}{l}300- \\
600^{\mathrm{b}}\end{array}$ & $\begin{array}{c}2- \\
250^{\mathrm{a}}\end{array}$ & --- \\
\hline
\end{tabular}

a- Kabata-Pendias and Pendieas (1992), b- ISI. (1983).

The studied elements are toxic with respect to the total form of cadmium element only in all sites at two seasons; and total iron in sites No. 1 and 3 in summer season, but the other elements were within the safe limits allowed exception $\mathrm{Cr}$ and $\mathrm{Ni}$ in sites No.1 and 3 in summer and winter season; according to (EU, 2002). The averages of total elements of soils in summer and winter seasons were $(37852.0,744.6,71.7,54.3,2.4,8.9,19.2,94.7,50.1$ and 17.6) and (40040.0, 660.8, 61.8, 46.7, 2.2, 7.4, 18.4, 82.7, 50.0 and 16.6) for $\mathrm{Fe}, \mathrm{Mn}, \mathrm{Zn}, \mathrm{Cu}, \mathrm{B}, \mathrm{Cd}, \mathrm{Co}, \mathrm{Cr}, \mathrm{Ni}$ and $\mathrm{Pb}$, respectively. 
J. Environ. Sci.

Institute of Environmental Studies and Research - Ain Shams University

Table (10): Assessment of total heavy metals in surface and sub-surface soils irrigated from El-Mariouteya canal.

\begin{tabular}{|c|c|c|c|c|c|c|c|c|c|c|c|}
\hline \multirow{3}{*}{ Sites No. } & \multirow{3}{*}{$\begin{array}{c}\text { Soil } \\
\text { depth } \\
(\mathbf{c m})\end{array}$} & \multicolumn{10}{|c|}{ Concentration $\mathrm{mg} \mathrm{kg}^{-1}$} \\
\hline & & Cd & Co & $\mathrm{Cr}$ & $\mathbf{N i}$ & $\mathbf{P b}$ & Cd & Co & $\mathbf{C r}$ & $\mathbf{N i}$ & $\mathbf{P b}$ \\
\hline & & \multicolumn{5}{|c|}{ Summer season } & \multicolumn{5}{|c|}{ Winter season } \\
\hline \multirow{2}{*}{1} & $0-20$ & 10.1 & 21.8 & 147.6 & 107.2 & 22.1 & 9.5 & 21.9 & 143.4 & 160.5 & 19.6 \\
\hline & $20-40$ & 6.5 & 21.6 & 67.4 & 57.0 & 21.1 & 5.8 & 21.0 & 41.2 & 41.1 & 18.4 \\
\hline \multirow{2}{*}{2} & $0-20$ & 6.5 & 17.6 & 121.1 & 59.7 & 20.9 & 6.3 & 17.0 & 117.2 & 53.0 & 20.1 \\
\hline & $20-40$ & 6.2 & 16.0 & 62.3 & 49.2 & 19.5 & 5.7 & 15.5 & 45.5 & 43.3 & 18.3 \\
\hline \multirow{2}{*}{3} & $0-20$ & 17.5 & 25.3 & 157.8 & 77.4 & 19.2 & 14.2 & 22.7 & 152.3 & 48.7 & 18.4 \\
\hline & $20-40$ & 15.2 & 20.9 & 122.3 & 52.1 & 16.1 & 13.5 & 19.6 & 114.9 & 38.4 & 14.4 \\
\hline \multirow{2}{*}{8} & $0-20$ & 12.2 & 19.9 & 96.1 & 42.8 & 16.4 & 8.4 & 18.0 & 62.0 & 35.0 & 15.3 \\
\hline & $20-40$ & 9.5 & 19.6 & 50.1 & 40.6 & 14.4 & 7.9 & 14.3 & 49.4 & 30.3 & 13.9 \\
\hline \multirow{2}{*}{10} & $0-20$ & 7.6 & 20.8 & 108.8 & 51.8 & 18.4 & 6.3 & 19.6 & 76.4 & 48.0 & 18.2 \\
\hline & $20-40$ & 7.4 & 18.1 & 64.8 & 42.6 & 16.0 & 5.5 & 17.2 & 62.8 & 45.4 & 15.6 \\
\hline \multirow{2}{*}{11} & $0-20$ & 6.6 & 19.3 & 130.4 & 45.9 & 18.7 & 5.9 & 18.3 & 92.4 & 33.7 & 16.7 \\
\hline & $20-40$ & 5.8 & 19.3 & 105.2 & 44.9 & 17.0 & 4.7 & 17.7 & 90.2 & 32.7 & 15.1 \\
\hline \multirow{2}{*}{15} & $0-20$ & 6.3 & 17.8 & 60.2 & 49.4 & 18.3 & 5.3 & 17.8 & 57.1 & 46.9 & 14.2 \\
\hline & $20-40$ & 6.4 & 16.1 & 57.2 & 44.4 & 15.9 & 4.5 & 16.6 & 53.2 & 42.3 & 14.2 \\
\hline \multicolumn{2}{|c|}{ Minimum } & 5.8 & 16.0 & 50.1 & 40.6 & 14.4 & 4.5 & 14.3 & 41.2 & 30.3 & 13.9 \\
\hline \multicolumn{2}{|c|}{ Maximum } & 17.5 & 25.3 & 157.8 & 77.4 & 20.9 & 14.2 & 22.7 & 152.3 & 160.5 & 20.1 \\
\hline \multicolumn{2}{|c|}{ Average } & 8.9 & 19.2 & 94.7 & 50.1 & 17.6 & 7.4 & 18.4 & 82.7 & 50.0 & 16.6 \\
\hline \multicolumn{2}{|c|}{ Critical limit } & $3^{\mathrm{a}}$ & -- & $150^{\mathrm{a}}$ & $75^{\mathrm{a}}$ & $300^{\mathrm{a}}$ & $3^{a}$ & -- & $150^{\mathrm{a}}$ & $75^{\mathrm{a}}$ & $300^{\mathrm{a}}$ \\
\hline
\end{tabular}

a- (EU, 2002).

6. Micro-nutrients and heavy metals contents in different plants irrigated for El-Mariouteya canal at different sites during summer and winter seasons: The irrigation with low quality water generally leads to change in chemical properties of soil and consequently micro-nutrients and heavy metals contents in growing plants. The concentrations of $\mathrm{Fe}, \mathrm{Mn}, \mathrm{Zn}, \mathrm{Cu}, \mathrm{B}$, 
$\mathrm{Cd}, \mathrm{Co}, \mathrm{Cr}, \mathrm{Ni}$ and $\mathrm{Pb}$ in plants grown in soils irrigated from at different sites during summer and winter seasons are showed in Table (11).

The $\mathrm{Pb}$ concentration in summer season in Okra fruits in site 2; Elephant forage in site 3; and Molokhia in site 15 were (3.00, 0.20, and $0.60 \mathrm{mg} \mathrm{kg}^{-1}$ ), respectively; whereas $\mathrm{Pb}$ was not found in all growing plants at winter season. It is clear from the previous results that cadmium not found in detectable concentrations in summer and winter seasons. Generally, the sequence of heavy metals in the studied plants were as follows $\mathrm{Fe}>\mathrm{Cr}>\mathrm{Ni}>\mathrm{Mn}>\mathrm{Zn}>$ $\mathrm{B}>\mathrm{Cu}>\mathrm{Co}$. In winter season results showed that the lowest concentration values were $\left(99.0 ; 1.9,0.20,41.1\right.$ and $16.5 \mathrm{mg} \mathrm{kg}^{-1}$ ) for $\mathrm{Fe}, \mathrm{B}, \mathrm{Co}, \mathrm{Cr}$ and $\mathrm{Ni}$ found in Wheat yield; and Cabbage were $26.60 \mathrm{mg} \mathrm{kg}^{-1}$ for $\mathrm{Mn} ; 12.9 \mathrm{mg} \mathrm{kg}^{-1}$ for $\mathrm{Zn}$ and $3.7 \mathrm{mg} \mathrm{kg}^{-1}$ for $\mathrm{Cu}$. While, the highest concentration values were (866.0 and $354.0 \mathrm{mg} \mathrm{kg}^{-1}$ ) for $\mathrm{Fe}$ and $\mathrm{Cr}$ were found in Onion; (71.6; 54.4 and $15.4 \mathrm{mg} \mathrm{kg}^{-1}$ ) for $\mathrm{Mn}, \mathrm{Zn}$ and $\mathrm{Cu}$ were found in Spinach; B was $18.3 \mathrm{mg} \mathrm{kg}^{-1}$ was found in Parsley; (1.50 and $129.1 \mathrm{mg} \mathrm{kg}^{-1}$ ) for $\mathrm{Co}$ and $\mathrm{Ni}$. 
J. Environ. Sci.

Institute of Environmental Studies and Research - Ain Shams University

Table (11): Assessment of micro nutrients and heavy metals in different plants grown in soil irrigated from El-Mariouteya canal.

\begin{tabular}{|c|c|c|c|c|c|c|c|c|c|c|c|c|}
\hline \multirow{2}{*}{ Season } & \multirow{2}{*}{$\begin{array}{l}\text { Site } \\
\text { No. }\end{array}$} & \multirow{2}{*}{$\begin{array}{c}\text { Plants } \\
\text { namely }\end{array}$} & \multicolumn{10}{|c|}{ Concentration $\mathrm{mg} \mathrm{kg}^{-1}$} \\
\hline & & & $\mathrm{Fe}$ & $\mathrm{Mn}$ & $\mathrm{Zn}$ & $\mathrm{Cu}$ & $\mathrm{B}$ & $\mathrm{Cd}$ & $\mathrm{Co}$ & $\mathrm{Cr}$ & $\mathrm{Ni}$ & $\mathrm{Pb}$ \\
\hline \multirow{7}{*}{ 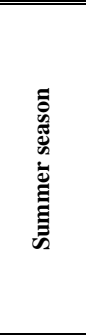 } & 1 & Arugula & 1257 & 58.0 & 94.0 & 44.0 & 22.0 & 0.00 & 0.80 & 328.0 & 80.4 & 0.00 \\
\hline & 2 & $\begin{array}{l}\text { Okra } \\
\text { fruits } \\
\end{array}$ & 5857 & 117.0 & 46.2 & 109.8 & 11.2 & 0.00 & 8.60 & 2256.0 & 719.4 & 3.00 \\
\hline & 3 & $\begin{array}{c}\text { Elephant } \\
\text { forage }\end{array}$ & 3476 & 77.6 & 57.0 & 63.6 & 8.8 & 0.00 & 3.80 & 1473.0 & 318.4 & 0.20 \\
\hline & 8 & $\begin{array}{c}\text { Elephant } \\
\text { forage }\end{array}$ & 1352 & 23.2 & 32.6 & 29.6 & 8.0 & 0.00 & 0.60 & 69.0 & 81.6 & 0.00 \\
\hline & 10 & $\begin{array}{l}\text { Eggplant } \\
\text { fruits }\end{array}$ & 535 & 13.2 & 30.8 & 20.8 & 15.2 & 0.00 & 0.00 & 44.0 & 6.0 & 0.00 \\
\hline & 11 & Maize & 519 & 44.6 & 15.9 & 13.5 & 3.8 & 0.00 & 0.60 & 241.0 & 69.9 & 0.00 \\
\hline & 15 & Molokhia & 4357 & 79.2 & 34.8 & 90.2 & 10.0 & 0.00 & 4.40 & 2272.0 & 483.8 & 0.60 \\
\hline \multicolumn{3}{|c|}{ Minimum } & 519 & 13.2 & 15.9 & 13.5 & 3.8 & 0.00 & 0.00 & 44.0 & 6.0 & 0.00 \\
\hline \multicolumn{3}{|c|}{ Maximum } & 5857 & 117.0 & 94.0 & 109.8 & 22.0 & 0.00 & 8.60 & 2272.0 & 719.4 & 3.00 \\
\hline \multicolumn{3}{|c|}{ Average } & 2479 & 59.0 & 44.5 & 53.1 & 11.3 & 0.00 & 2.69 & 954.7 & 251.4 & 0.54 \\
\hline \multirow{7}{*}{ 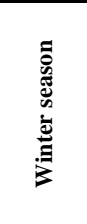 } & 1 & Cabbage & 516 & 32.8 & 23.2 & 9.0 & 16.5 & 0.00 & 0.30 & 138.0 & 30.0 & 0.00 \\
\hline & 2 & Parsley & 496 & 30.9 & 26.1 & 14.2 & 18.3 & 0.00 & 0.80 & 153.0 & 40.9 & 0.00 \\
\hline & 3 & Wheat & 99 & 29.1 & 18.5 & 4.9 & 1.9 & 0.00 & 0.20 & 41.0 & 16.5 & 0.00 \\
\hline & 8 & Mallow & 649 & 44.3 & 42.7 & 11.7 & 11.4 & 0.00 & 0.50 & 90.0 & 42.0 & 0.00 \\
\hline & 10 & Onion & 866 & 41.3 & 22.5 & 10.6 & 5.6 & 0.00 & 1.20 & 354.0 & 76.6 & 0.00 \\
\hline & 11 & Spinach & 515 & 71.6 & 54.5 & 15.4 & 14.2 & 0.00 & 0.90 & 203.0 & 72.4 & 0.00 \\
\hline & 15 & Cabbage & 478 & 26.6 & 12.9 & 3.7 & 3.8 & 0.00 & 1.50 & 341.0 & 129.1 & 0.00 \\
\hline \multicolumn{3}{|c|}{ Minimum } & 99 & 26.6 & 12.9 & 3.7 & 1.9 & 0.00 & 0.20 & 41.0 & 16.5 & 0.00 \\
\hline \multicolumn{3}{|c|}{ Maximum } & 866 & 71.6 & 54.5 & 15.4 & 18.3 & 0.00 & 1.50 & 354.0 & 129.1 & 0.00 \\
\hline \multicolumn{3}{|c|}{ Average } & 517 & 39.5 & 28.6 & 9.9 & 10.2 & 0.00 & 0.77 & 188.6 & 58.2 & 0.00 \\
\hline \multicolumn{3}{|c|}{ Critical levels* } & $50-250$ & $20-300$ & $20-50$ & $5-20$ & -- & $0.02-1.2$ & -- & -- & $0-4$ & $0.1-30$ \\
\hline
\end{tabular}

- Bennett (1993), Adriano (1986); Misra and Mani (1991).7. Bio concentration factor $(\mathrm{BCF})$ :

Plants may represent an important source of elements for humans as it is well known that metals in soil may be taken up by plants and enter the food chain. The BCR of different plants tissues grow in soil irrigated from ElMariouteya canal at different sites during summer and winter seasons are presented in Table (12). Data showed that, each plant has specified capability to accumulate elements in their tissue i.e. generally all plants were hyperaccumulator for (Fe, Mn, $\mathrm{Zn}, \mathrm{B}, \mathrm{Co}, \mathrm{Cr}$ and $\mathrm{Ni}$ ) at summer and winter seasons except Eggplant fruits with $\mathrm{Co}$ and Wheat plant with $\mathrm{Cu}$ were influences. As well as this the BCF for all plants with $\mathrm{Cd}$ and $\mathrm{Pb}$; the plants can be an 
excluder except with Okra fruits and Molokhia. This behavior could be attributed to one or more of the following processes: (1) plant adsorb heavy metals, translocate them through tonoplast and accumulate in vacuoles, thereby, protecting cell metabolism from metal toxicity, Sekar et al., (2004), (2) binding of the cationic element form to the anionic sites in the cell wall Zhu et al., (1999), (3) binding to non-proteinaceous polypeptides (Phyto 12 chelations) and accumulate in the vacuole Sacchi et al., (1999). The advantage of high biomass productive and easy disposal makes plants most useful to remediate heavy metals on site. Based on knowledge of the heavy metal accumulation in plants, it is possible to select those species of crops and pasturage herbs, which accumulate fewer heavy metals, for food cultivation and fodder for animals, and to select those hyper accumulation species for extracting heavy metals from soil and water. 
J. Environ. Sci.

Institute of Environmental Studies and Research - Ain Shams University

Table (12): The BCR of different plants tissues grow in soil irrigated from El-Mariouteya canal.

\begin{tabular}{|c|c|c|c|c|c|c|c|c|c|c|c|}
\hline \multirow{2}{*}{ Sites No. } & \multirow{2}{*}{$\begin{array}{l}\text { Plants } \\
\text { namely }\end{array}$} & \multicolumn{10}{|c|}{ Concentration mg kg ${ }^{-1}$} \\
\hline & & $\mathrm{Fe}$ & $\mathrm{Mn}$ & $\mathrm{Zn}$ & $\mathrm{Cu}$ & B & $\mathrm{Cd}$ & Co & $\mathrm{Cr}$ & $\mathrm{Ni}$ & $\mathrm{Pb}$ \\
\hline \multicolumn{12}{|c|}{ Summer season } \\
\hline 1 & Arugula & 51.8 & 7.6 & 25.0 & 6.6 & 220.0 & 0.0 & 20.0 & 16400.0 & 277.2 & 0.0 \\
\hline 2 & Okra fruits & 457.2 & 9.4 & 12.5 & 23.0 & 62.2 & 0.0 & 78.2 & 112800.0 & 1308.0 & 1.8 \\
\hline 3 & $\begin{array}{l}\text { Elephant } \\
\text { forage }\end{array}$ & 187.7 & 11.0 & 16.2 & 10.3 & 40.0 & 0.0 & 63.3 & 73650.0 & 860.5 & 0.2 \\
\hline 8 & $\begin{array}{l}\text { Elephant } \\
\text { forage }\end{array}$ & 55.0 & 2.2 & 6.5 & 5.1 & 100.0 & 0.0 & 10.0 & 1380.0 & 189.8 & 0.0 \\
\hline 10 & $\begin{array}{l}\text { Eggplant } \\
\text { fruits }\end{array}$ & 10.1 & 1.5 & 3.2 & 1.7 & 126.7 & 0.0 & 0.0 & 2200.0 & 15.0 & 0.0 \\
\hline 11 & Maize & 16.4 & 4.0 & 1.9 & 1.8 & 34.5 & 0.0 & 8.6 & 8033.3 & 92.0 & 0.0 \\
\hline 15 & Molokhia & 260.6 & 10.4 & 14.8 & 17.9 & 250.0 & 0.0 & 220.0 & 227200.0 & 2015.8 & 1.4 \\
\hline \multicolumn{12}{|c|}{ Winter season } \\
\hline 1 & Cabbage & 29.6 & 5.3 & 6.9 & 1.5 & 183.3 & 0.0 & 10.0 & 13800.0 & 120.0 & 0.0 \\
\hline 2 & Parsley & 51.6 & 3.5 & 7.9 & 3.0 & 140.8 & 0.0 & 8.9 & 15300.0 & 120.3 & 0.0 \\
\hline 3 & Wheat & 6.2 & 3.6 & 6.4 & 1.0 & 9.5 & 0.0 & 4.0 & 2050.0 & 48.5 & 0.0 \\
\hline 8 & Mallow & 65.1 & 4.3 & 11.1 & 2.2 & 142.5 & 0.0 & 12.5 & 2250.0 & 105.0 & 0.0 \\
\hline 10 & Onion & 18.3 & 5.1 & 2.7 & 1.1 & 93.3 & 0.0 & 24.0 & 17700.0 & 117.8 & 0.0 \\
\hline 11 & Spinach & 25.5 & 9.2 & 17.0 & 2.3 & 177.5 & 0.0 & 22.5 & 10150.0 & 206.9 & 0.0 \\
\hline 15 & Cabbage & 30.0 & 6.5 & 7.0 & 1.1 & 126.7 & 0.0 & 75.0 & 34100.0 & 445.2 & 0.0 \\
\hline
\end{tabular}

\section{Contamination Factors; Degree of Contamination, Modified Degree of}

\section{Contamination and Pollution Load Index:}

Soils irrigated from (El-Moheet and El-Rahawy drains) are assessed for contamination factors $(\mathrm{CF})$, degree of contamination $(\mathrm{Cd})$, modified degree of contamination $(\mathrm{mCd})$ and the pollution load index (PLI). The results are shown in Table (13). It is obvious from these results that CF values indicated that soils were low in $(\mathrm{CF})$ with $\mathrm{Zn}, \mathrm{B}$ and $\mathrm{Pb}$ for all sites; moderately $(\mathrm{CF})$ with $\mathrm{Cu}$ and $\mathrm{Co}$ in all sites, low to moderate (CF) with $\mathrm{Fe}, \mathrm{Mn}, \mathrm{Cr}$ and $\mathrm{Ni}$; 
low in $(\mathrm{CF})$ for Fe in sites 2, 8, 11 and 15; and moderate $(\mathrm{CF})$ at summer season in sites 1, 3 and 10. As well as in winter season was low (CF) in all sites except sites 1 and 3. With regard to Mn was a moderate (CF) in all sites except site 2 was low (CF) at summer; but in winter season was low (CF) in sites 2,11 and 15 only and was moderate in sites 1, 3, 8 and 10. Concerning contamination factor for $\mathrm{Cr}$ was low $(\mathrm{CF})$ in sites (2, 8 and 11) and (1 and 3) at summer and winter seasons respectively and other sites were moderately $(\mathrm{CF})$. With regard to $\mathrm{Ni}$ was low $(\mathrm{CF})$ in sites $(8,10,11$ and 15); and moderate $(\mathrm{CF})$ in sites 1,2 and 3 at summer season; while in winter season was low (CF) for all sites except site 1 was moderate; but showed very high (CF) with $\mathrm{Cd}$. The contamination factor for the different metals generally followed the sequence $\mathrm{Cd}>\mathrm{Cu}>\mathrm{Fe}>\mathrm{Ni}>\mathrm{Co}>\mathrm{Mn}>\mathrm{Cr}>\mathrm{Pb}>\mathrm{Zn}>\mathrm{B}$. On the other hand, in winter season it can be concluded form these results that the highest contamination was for $\mathrm{Cd}$ in site 3 in winter and summer seasons.

In case of degree of contamination (Cd), soils at summer season fall under considerable $(\mathrm{Cd})$ in sites $(2,10,11$ and 15) and very high $(\mathrm{Cd})$, indicating serious anthropogenic pollution in sites (1, 3 and 8); also (Cd), soils at winter season was fall under considerable $(\mathrm{Cd})$ in all sites except sites (1 and 3) was very high (Cd), indicating serious anthropogenic pollution. The $(\mathrm{mCd})$ suggested that the studied soil showed moderate degree of $(\mathrm{mCd})$ in sites $(1,2,10,11$ and 15) and high degree of $(\mathrm{mCd})$ in sites (3 and 8) at summer season; while in winter season, the values of $\left({ }_{\mathrm{m}} \mathrm{Cd}\right)$ varied from 2.166 to 4.880 . These values indicating moderate degree of $(\mathrm{mCd})$ in all studied sites except site 3 showed high degree of $(\mathrm{mCd})$. 
Pollution severity and its variation along the sites were determined with the use of pollution load index. This index is used to compare pollution status of different places (Tomlinson et al., 1980). In summer season, the values of pollution load index are found to be generally high $(>1)$ in all sites except site 15 was moderate (0.948). Consequently, the pollution load index suggested deterioration of site quality in all sites except site 15 showed base line pollution level of pollution level. These results confirmed that long term irrigation with polluted water might increase the accumulation of heavy metals in soil. Whereas, the values of PLI in winter season are found to be low $(<1)$ in sites $(2,8,11$ and 15$)$, these values indicating perfection; whereas, and other sites (1, 3 and 10) showed PLI equal to 1. 
Taha, Aya, et al

Table (13): Contamination factor, contamination degree, and modified degree of contamination and pollution load index of heavy metals in soil at El-

Mariouteya canal during summer and winter seasons.

\begin{tabular}{|c|c|c|c|c|c|c|c|c|c|c|c|c|c|c|}
\hline \multirow{2}{*}{ 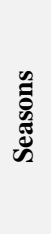 } & \multirow{2}{*}{ 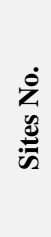 } & \multicolumn{10}{|c|}{ Concentration of contamination factor $\mathrm{mg} \mathrm{kg}^{-1}$} & \multirow{2}{*}{ 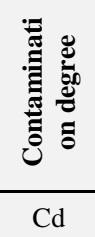 } & \multirow{2}{*}{ 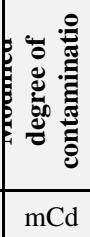 } & \multirow{2}{*}{ 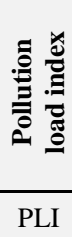 } \\
\hline & & $\mathrm{Fe}$ & $\mathrm{Mn}$ & $\mathrm{Zn}$ & $\mathrm{Cu}$ & B & $\mathrm{Cd}$ & Co & $\mathrm{Cr}$ & $\mathrm{Ni}$ & $\mathrm{Pb}$ & & & \\
\hline \multirow{7}{*}{ 离 } & 1 & 1.98 & 1.37 & 0.49 & 1.84 & 0.22 & 28.056 & 1.46 & 1.21 & 1.88 & 0.925 & 39.436 & 3.944 & 1.466 \\
\hline & 2 & 0.84 & 0.99 & 0.45 & 1.68 & 0.14 & 18.056 & 1.18 & 0.99 & 1.05 & 0.874 & 26.252 & 2.625 & 1.053 \\
\hline & 3 & 1.58 & 1.36 & 0.50 & 2.39 & 0.19 & 48.611 & 1.70 & 1.29 & 1.36 & 0.803 & 59.784 & 5.978 & 1.498 \\
\hline & 8 & 0.97 & 1.20 & 0.47 & 1.92 & 0.14 & 33.889 & 1.34 & 0.79 & 0.75 & 0.686 & 42.133 & 4.213 & 1.100 \\
\hline & 10 & 1.05 & 1.27 & 0.61 & 2.37 & 0.14 & 21.111 & 1.40 & 0.89 & 0.91 & 0.770 & 30.512 & 3.051 & 1.175 \\
\hline & 11 & 0.94 & 1.24 & 0.60 & 2.24 & 0.13 & 18.333 & 1.30 & 1.07 & 0.81 & 0.782 & 27.436 & 2.744 & 1.127 \\
\hline & 15 & 0.96 & 1.50 & 0.51 & 1.61 & 0.07 & 17.500 & 1.20 & 0.49 & 0.87 & 0.766 & 25.466 & 2.547 & 0.948 \\
\hline \multirow{7}{*}{ 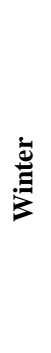 } & 1 & 1.91 & 1.09 & 0.45 & 1.80 & 0.21 & 26.389 & 1.47 & 1.18 & 2.82 & 0.820 & 38.125 & 3.812 & 1.433 \\
\hline & 2 & 0.74 & 0.92 & 0.42 & 1.65 & 0.12 & 17.500 & 1.14 & 0.96 & 0.93 & 0.841 & 25.225 & 2.523 & 0.981 \\
\hline & 3 & 1.45 & 1.14 & 0.41 & 1.81 & 0.15 & 39.444 & 1.52 & 1.25 & 0.85 & 0.770 & 48.800 & 4.880 & 1.249 \\
\hline & 8 & 0.92 & 1.12 & 0.45 & 1.55 & 0.11 & 23.333 & 1.21 & 0.51 & 0.61 & 0.640 & 30.451 & 3.045 & 0.923 \\
\hline & 10 & 0.99 & 1.26 & 0.59 & 1.75 & 0.12 & 17.500 & 1.32 & 0.63 & 0.84 & 0.762 & 25.759 & 2.576 & 1.033 \\
\hline & 11 & 0.90 & 0.90 & 0.43 & 1.51 & 0.08 & 16.389 & 1.23 & 0.76 & 0.59 & 0.699 & 23.490 & 2.349 & 0.880 \\
\hline & 15 & 0.91 & 0.98 & 0.38 & 1.52 & 0.07 & 14.722 & 1.20 & 0.47 & 0.82 & 0.594 & 21.661 & 2.166 & 0.823 \\
\hline
\end{tabular}

\section{REFERENCES}

Abrahim, G. and Parker, R. (2008). Assessment of heavy metal enrichment factors and the degree of contamination in marine sediments from Tamaki Estuary. Auckland, New Zealand. Environmental Monitoring and Assessment, 136 (1-3): 227 - 238.

Abrahim, G. M. S. (2005). Holocene sediments of Tamaki Estuary, characterization and impact of recent human activity on an urban estuary in Auckland, New Zealand, PhD Thesis Univ. of Auckland: Auckland, New Zealand, 5 - 275. P361. 
Adepetu, J. A.; Nabhan, H. and Osinubi, A. (1996). Simple soil, water and plant testing techniques for soil resource management. Proceedings of a training course held in Ibadan, Nigeria, 16-27.

Adriano, D. C. (1986). Trace elements in the Terrestrial environment. Spring - Verlage.

Ali, M. M.; Ali, M. L.; Islam, S. and Rahman, Z. (2016). Preliminary assessment of heavy metals in water and sediment of karnaphuli river, Bangladesh. Envir. Nanotechn., Monit. and Manag., 5: 2735 .

Ayers, R. S. and Westcot, D. W. (1985). Water quality for agriculture. FAO Irrigation and drainage paper 29 Rev. 1. Food and Agric. Org. Rome, 1,74 .

Balkhair, K. S. and Ashraf, M. A. (2016). Field accumulation risks of heavy metals in soil and vegetable crop irrigated with sewage water in western region of Saudi Arabia. Saudi J. of biological sciences, 23(1): 32-44.

Bennett, W. F. (1993). Nutrient deficiencies and toxicities in crop plants. College of Agric. Sci. Nautural Resources, Texas Tech Univ., Lubbock, Berlin, Heidelberg, New York, p.536.

Bradford, G. R.; Change, A. C.; Page, A. L.; Bakhtar, D.; Frapton, J. A. and Wright, H. (1996). Background concentrations of Trace and Major Elements in California Soils. Dr. Andrew C. Chang, Department of Environmental Sciences University of CA, Riverside, CA 92521.p 1-32.

Cottenie, A.; Verloo, M.; Kiekens, 1.; Velghe, G. and Camerlynck, R. (1982). Chemical analysis of plants and soils. Lab. of Analyt. and Agro. State, Univ. Ghent. Belgium.

Ekengele, L. N.; Blaise, A. and Jung, M. C. (2017). Accumulation of heavy metals in surface sediments of lere lake, Chad. Geosciences Journal, 21(2): 305-315. 
El-Tohamy, S. A.; Mahmoud, Y. I.; Afifi, M.; Wafaa, M. I. and Hafez, A. (2015). Environmental impact of using low quality water in irrigation. J. Soil Sci. and Agric. Eng., Mansoura Univ., 6 (9): $1029-1052$.

El-Kholy, M. M., Sherif, A. E. A., Mahmoud, Y., I. and El-Sayed, G. A. M. (2015). Evaluation of the contamination caused by human activities on EL-Zomor canal, EL-Giza Governorate. J.Soil Sci. and Agric. Eng., Mansoura Univ., 6(2): 323 - 336.

Elrashidi, M. A.; Mays, M. D. and Lee, C. W. (2003). Assessment of Mehlich3 and Ammonium Bicarbonate-DTPA Extraction for Simultaneous Measurement of Fifteen Elements in Soils. Communications in Soil Science and Plant Analysis 34 (19-20).

Ene, A.; Popescu, I. V. and Stihi, C. (2009). Applications of proton-induced $\mathrm{X}$-ray emission technique in materials and environmental science. Ovidius Univ Ann Chem, 20(1): 35-39.

EPA Guidelines (2007). Regulatory monitoring and testing water and wastewater sampling.

EPA. (1991). Methods for the Determination of Metals in Environmental Samples. Office of research and development Washington DC 20460 pp. 23 - 29 and $83-122$.

European Union standards, (EU). (2002). Heavy metals in wastes, European commission on environment.

FAO. (1985). Water quality for agriculture. Paper No. 29 (Rev. 1) UNESCO, Publication, Rome Italy.

FAO. (1992). Wastewater Treatment and use in Agriculture. Pescod MB. Irrigation and Drainage Paper 47. Food and Agricultural Organization (FAO), Rome.

Fatih, M. K.; Metin, T.; Ustun, S.; Ilker, A. and Omer, A. (2007). Effects of wastewater irrigation on soil and Cabbage-plant (Brassica olerecea var. capitate cv. yalova-1) chemical properties and Mustafa Okuroglu1 J. Plant Nutr. Soil Sci., 170: 166-172. 
Fytianos, K.; Katsianis, G.; Triantafyllou, P. and Zachariadis, G. (2001). Accumulation of heavy metals in vegetables grown in an industrial area in relation to soil. Bulletin of environmental contamination and toxicology, 67(3): 0423-0430.

Gaber, H. S.; El-Kasheif, M. A.; Ibrahim S. A. and Authman M. M. N. (2013). Effect of Water Pollution in El-Rahawy Drainage Canal on Hematology and Organs of Freshwater Fish Clarias gariepinus. World Appl. Sci. J., 21 (3): 329-341.

Hakanson, L. (1980). Ecological risk index for aquatic pollution control: sediment logical approach, Water Res., 14: 975-1001.

ICARDA "International Center for Agricultural Research in the Dry Areas" (2013). Methods of soil, Plant, and water analysis: A manual for the West Asia and North Africa region. Estefan, G., Sommer, R. and Ryan, J. $3^{\text {th }}$ edition. Box 114/5055, Beirut, Lebanon.

ISI (Indian Standard Institution) (1983). Specifications for drinking and irrigation waters. IS: 10500. New Delhi, India.

Kabata-Pendias and Pendieas (1992). Trace elements in soil and plants-CRC Press. inc., Boca Raton, Florida.

Kapourchal, S. A.; Pazira, E. and Homaee, M. (2009). "Assessing radish (Raphanus sativus L.) potential for phytoremediation of leadpolluted soils resulting from air pollution." Plant Soil Environ 55(5): 202-206.

Khan, H. A.; Arif, I. A. and Al Homaidan, A. A. (2012). Distribution pattern of eight heavy metals in the outer and inner tissues of ten commonly used vegetables. Intern. J. of food properties, 15(6): 1212-1219.

Kiziloglu, F.; Turan, M.; Sahin, U.; Angin, I.; Anapali, O. and Okuroglu, M. (2007). Effects of wastewater irrigation on soil and Cabbage-plant (brassica olerecea var. capitate cv. yalova-1) chemical properties. Journal of plant nutrition and soil science, 170(1): 166172. 
Lei, M.; Liao, B. H.; Zeng, Q. R.; Qin, P. F. and Khan, S. (2008). Fraction Distributions of Lead, Cadmium, Copper, and Zinc in Metal Contaminated Soil before and after Extraction with Disodium Ethylenediaminetetraacetic Acid. Communications in soil science and plant analysis, 39 (13-14): 1963 - 1978.

Likuku, A. S.; Mmolawa, K. B. and Gaboutloeloe, G. K. (2013). Assessment of heavy metal enrichment and degree of contamination around the copper-nickel mine in the selebi phikwe region, eastern Botswana. Environment and Ecology. Res.1 (2): 32 - 40.

Liu, W. H.; Zhao, J. Z.; Ouyang, Z. Y.; Soderlund, L. and Liu, G. H. (2005). Impacts of sewage irrigation on heavy metal distribution and contamination in Beijing, China. Environment International, 31 (6): $805-812$

Liu, W. X; Li, H. H.; Li, S. R. and Wang, Y. W. (2006). Heavy metal accumulation of edible vegetables cultivated in agricultural soil in the suburb of Zhengzhou City, People's Republic of China. Bulletin of Environmental Contamination and Toxicology, 76: 163-170.

Michael, C. A., O’Neil, K. P. and Perry, C. H. (2007). Soil vital signs: A new Soil Quality Index (SQI) for assessing forest soil health. Res. Pap. RMRS-RP-65WWW. Fort Collins, CO: U.S. Department of Agriculture, Forest Service, Rocky Mountain Research Station. $12 \mathrm{p}$.

Misra, S. G. and Mani, D. (1991). Soil Pollution. Ashish Publishing House, New Delhi, India.

Mohamed, M. S. (2014). Geophysical and geochemical method in the detection of ground water contamination zones, El-Minia district, Egypt. M.S. degree, Faculty of Science Minia University.

Mustapha, H. I. and Adeboye, O. B. (2014). Heavy metals accumulation in edible part of vegetables irrigated with untreated municipal wastewater in tropical savannah zone, Nigeria. African Journal of Environment Science and Technology, 8(8): 460-463. 
Omotoso, O. A. and Ojo, O. J. (2015). Assessment of some heavy metals contamination in the soil of river Niger floodplain at Jebba, central Nigeria. Water Utility J., 9: 71-80.

RMRS "Rocky Mountain Research Station" (2012). Sampling Procedure for Lake or Stream Surface Water Chemistry. United States Department of Agric., Forest Service, Rocky Mountain Research Station, Res. Note RMRS-RN - 49.

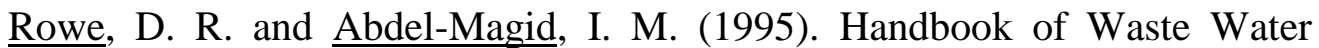
Reclamation and Reuse. Edition: 1 Publisher: CRC PressLewis Publishers.

Rusan, M. J. M.; Hinnawi, S. and Rousan, L. (2007). Long term effect of wastewater irrigation of forage crops on soil and plant quality parameters. Desalination, 215(1): 143-152.

Sacchi, G. A.; Rivetta, A.; Coucci, M.; Capri, E.; Boccelli, R.; Loffi, S. and Lombi, E. (1999). Radical absorption and bioaccumulation of heavy metals in plants: problems and prospects. Impatto. ambientale. metallic. pesanti-ed- element in trace: p. 65-76.

Sekar, K. C.; Chary, N. S.; Kamala, C. T. and Amjaneyulu, Y. (2004). Utilization of plant metal interactions for environmental management. Proceedings of Indian National Science Academy, Part B, Reviews and Tracts Biological Science. 70: 1 pp. 13-30.

Sherif, A. E. A.; El-Kholy, M. M. and Salem, T. M. (2015). Risk assessment of trace elements toxicity through contaminated edible plants from polluted irrigation canal at Giza governorate, Egypt. Iranica J. of Energy and Environment. 6 (1): 47 - 55.

Singh, A.; Sharma, R. K.; Agrawal, M. and Marshall, F. M. (2010). Health risk assessment of heavy metals via dietary intake of foodstuffs from the wastewater irrigated site of a dry tropical area of India. Food and Chem. Toxic., 48(2): 611-619.

Soltanpour, P. N. and Schwab, A. P. (1991). Determination of nutrient availability element toxicity by AB-DTPA. Soil Test ICPS Adv. Soil Sci., 16: 165 - 190. 
Tang, W. Z.; Zhao, Y.; Wang, C.; Shan, B. Q. and Cui, J. G. (2013). Heavy metal contamination of overlying waters and bed sediments of Haihe Basin in China. Ecotox. Environ. Safe. 98: 317-323.

Tomlinson, D.; Wilson, J.; Harris, C. and Jeffrey, D. (1980). Problems in the assessment of heavy-metal levels in estuaries and the formation of a pollution index. Helgolander Meeresuntersuchungen, 33 (1 - 4): $566-575$.

Turekian, K. K. and Wedepohl, K. H. (1961). Distribution of elements in some major units of the earth's crust. Geological Society of America, Bulletin. 72: 175-192.

Zhu, Y. L.; Zayed, A. A. M.; Qian, J. H.; Desoura, M. and Teery, N. (1999). Phytoaccumulation of trace elements by wetland plants: 11 . water hyacinth. J. Environ. Qual. 23: 339-344.

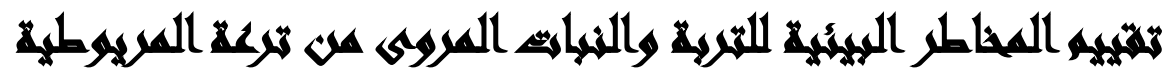

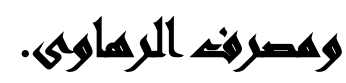

\section{[1]}

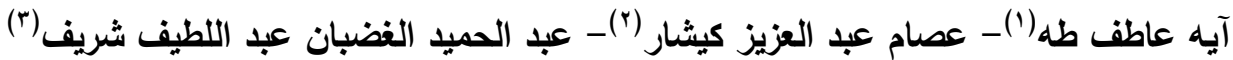
() جهاز شئون البيئة r) كلية البنات، جامعة عين شمس r) معهد بحوث الأراضى والمياه والبيئة

\section{المستخلص}

لقد أجريت هذه الدراسة بهدف تقييم المخاطر البيئية للمياهوالتربة والنباتات التى تروى من ترعة

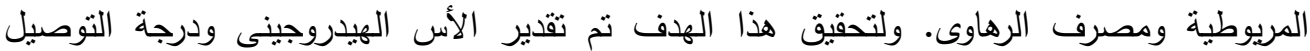

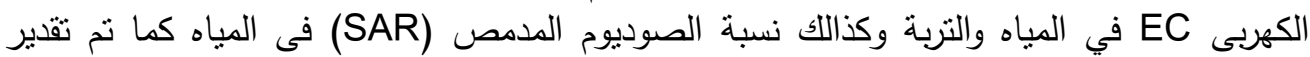

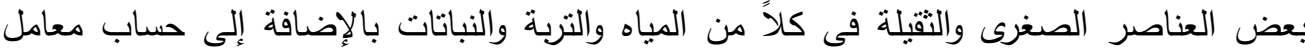

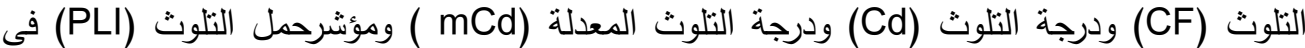

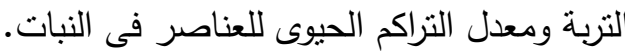

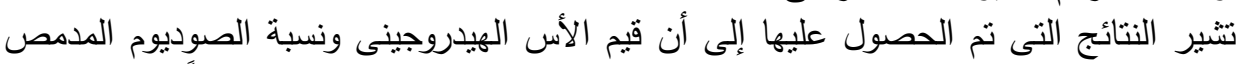

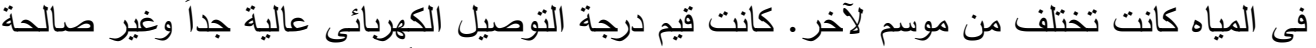
للرى في فصل الصيف بينما كانت صالحة للرى فى فصل الثتاء طبقاً لمنظمة الأغذية والزرعة. 
J. Environ. Sci.

Institute of Environmental Studies and Research - Ain Shams University

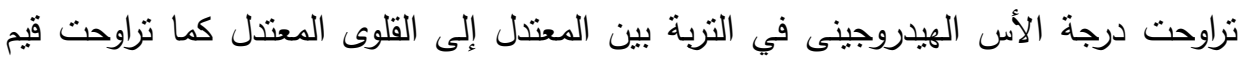

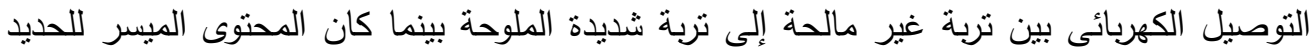

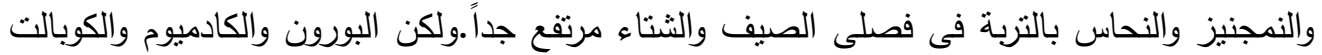

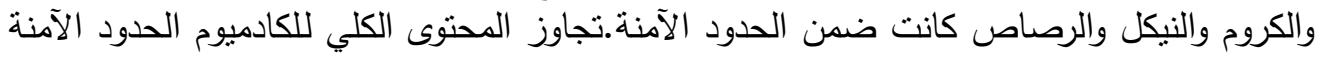

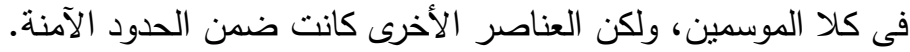

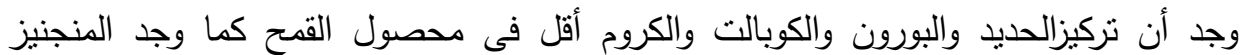

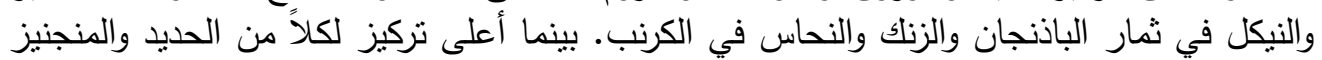

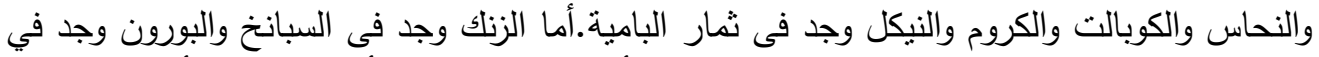

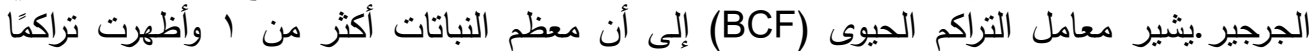
عاليًا للعناصر الثقيلة.

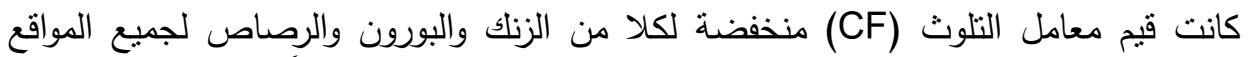

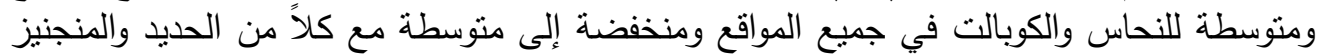

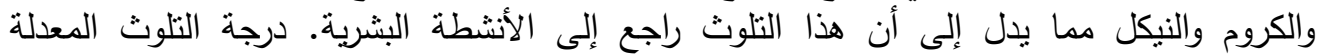
(mCd)

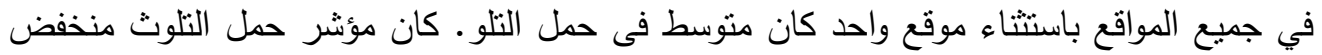
(1>) في بعض المواقع فى فصل الثناء الثناء. الكلمات المفتاحية: المياه، التربة، النبات، التلوث، تقييم مخاطر التراكم الحيوى. 